\title{
أستعمال أنموذج le'vy في تقدير عوائد الأسهم لبعض المصارف المارف العراقيت
}

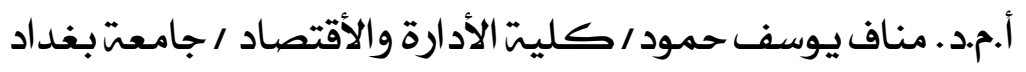
الباحث/ مريـم جمعهد موسى موسى

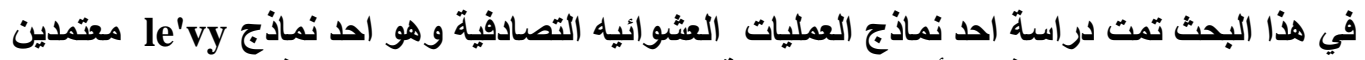

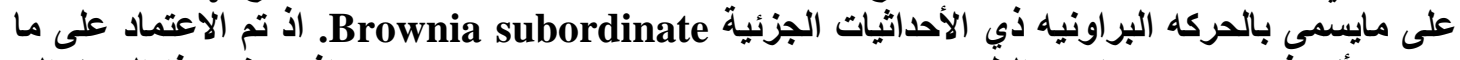

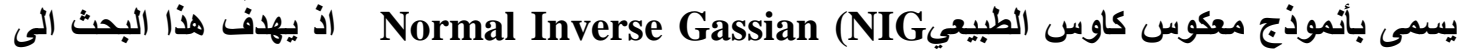

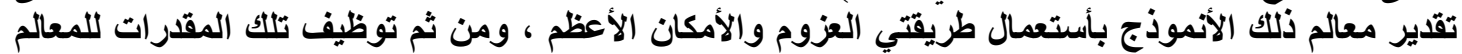

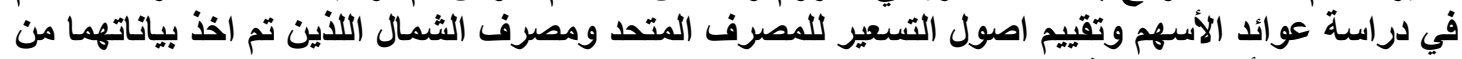

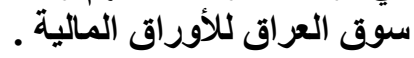
وقد تم التوصل الى النتيجة التي ترى التضلية مقدر الأمكان الأعظم على مقدر العزوم بالأعتماد على

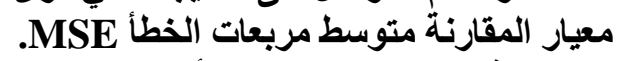

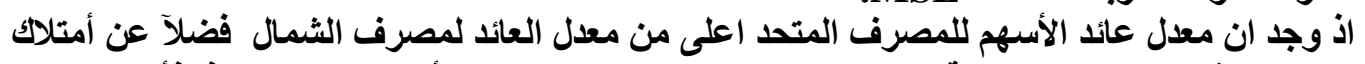

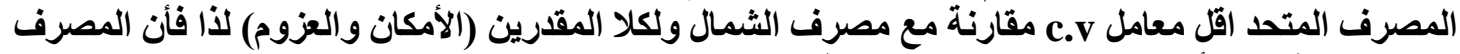

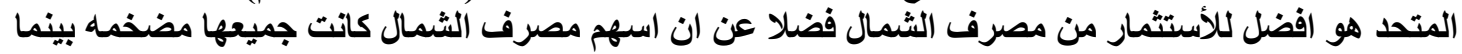

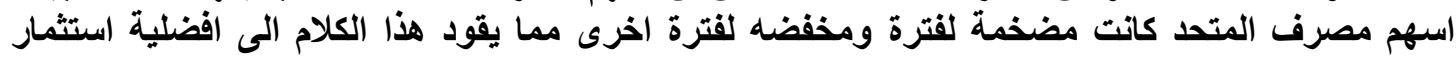

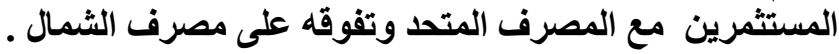

الإسطات الرئيسيس للابحث : الحركة البراونية، الأحداثي الجزئي، الأحداثي الجزئي ذو الحركة البراونية، أنموذج معكوس كاوس الطبيعي .

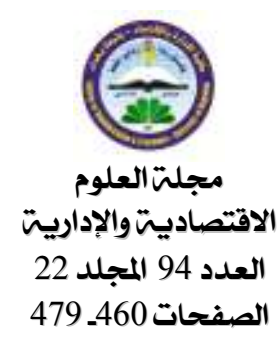




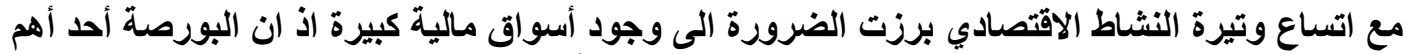

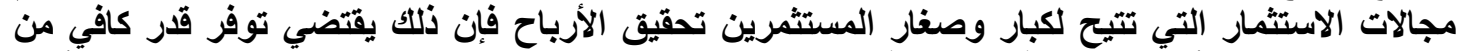

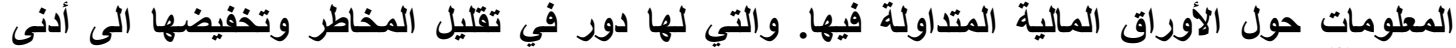

لذا فإن أساس العملية الاستثمارية هو تحليل الأسواق والأوراق المالية وكافة البدائل الاستثمارية من

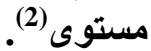

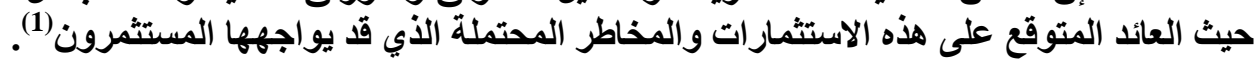

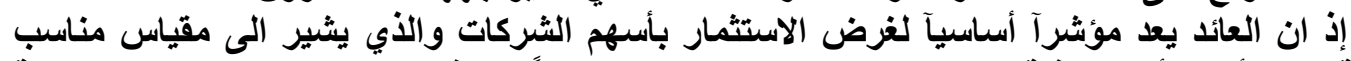

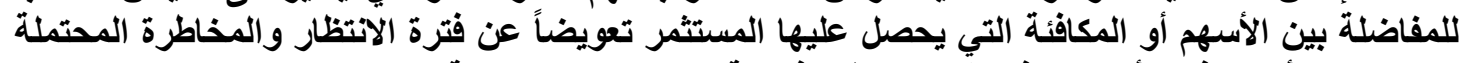

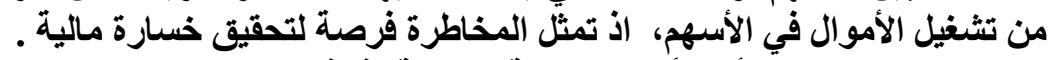

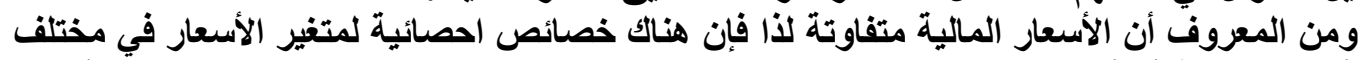

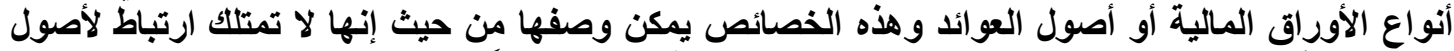

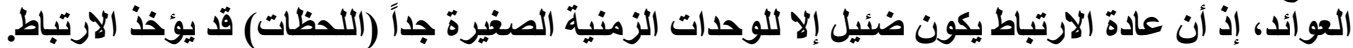

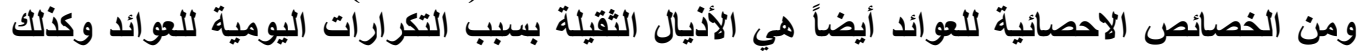

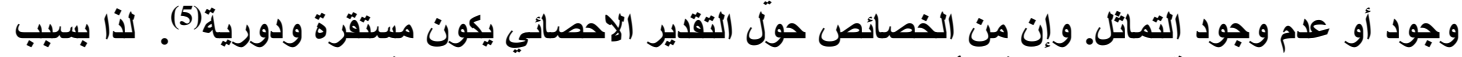

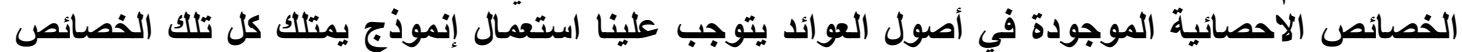

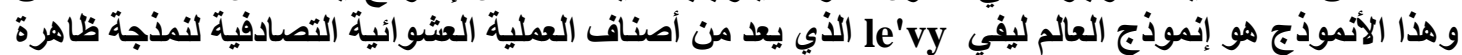

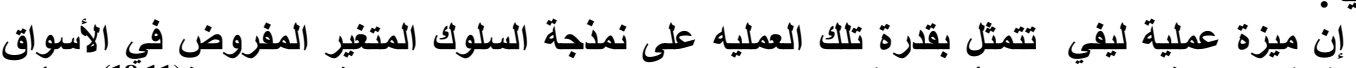
عشوائية.

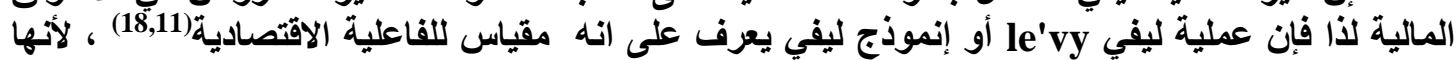

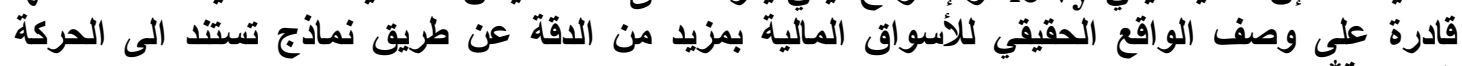
(البراونية" (Brownan Motion)

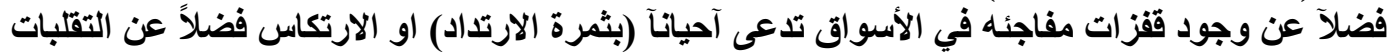

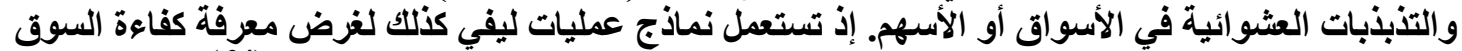

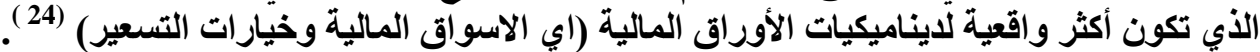

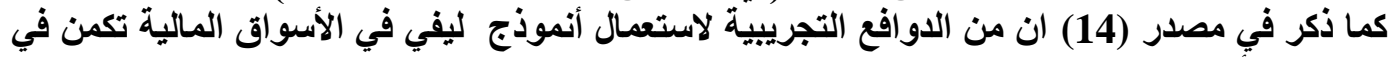
ملائمته لتوزيع أَصول العوائد.

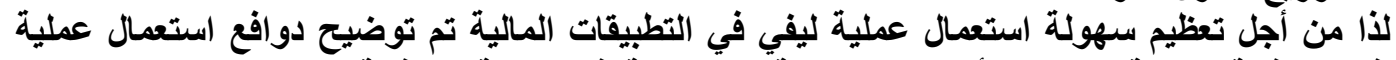

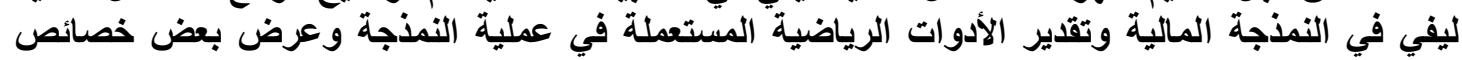

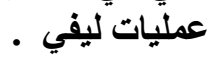

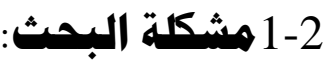

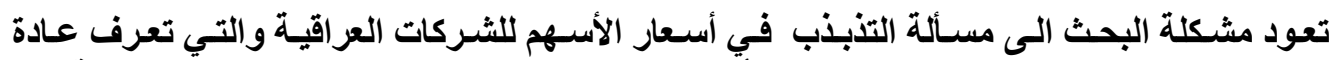

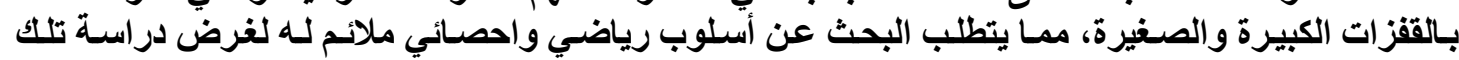

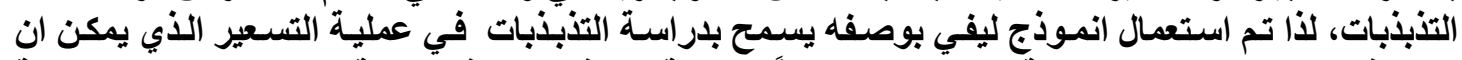

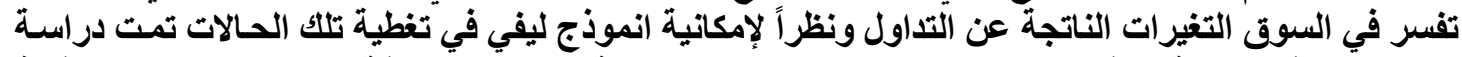

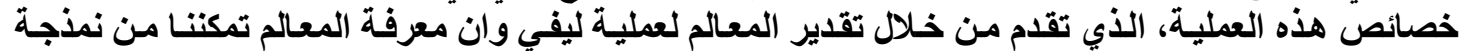

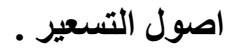

* حاله خاصه من عملية le'vy* بأفتراض أن المتغير العشوائي يتوزع توزيعاً طبيعياً 


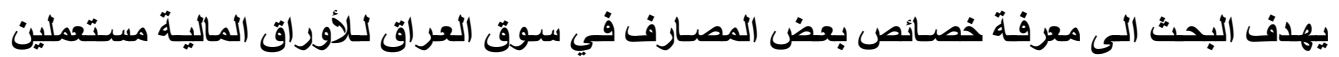

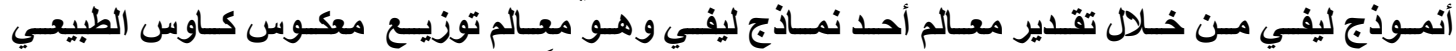

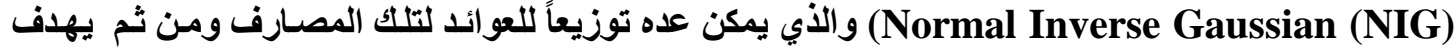

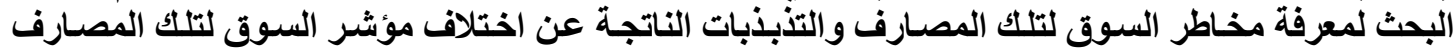

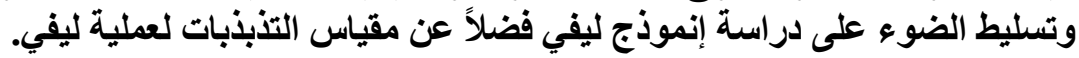

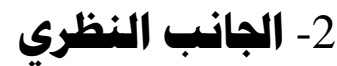

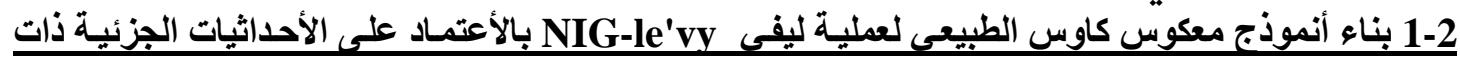

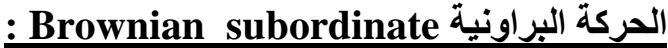

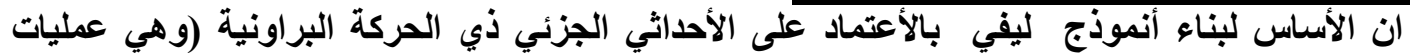

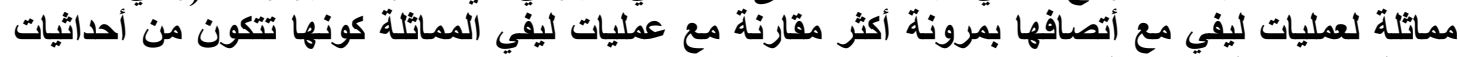

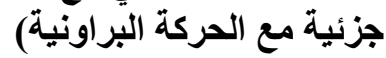

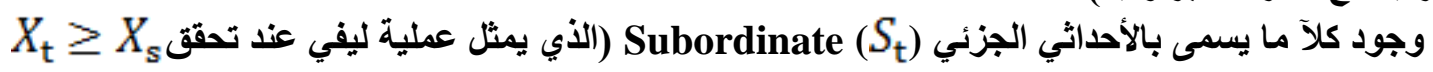

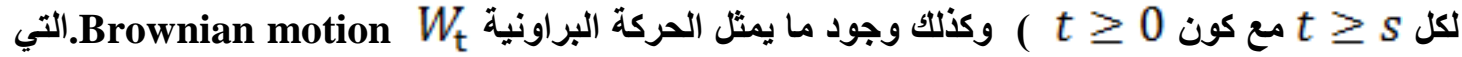
تكون مستقلة عن الأحداثي الجزئي (6) وفقآ للصيغيه الأتيه

$$
\begin{aligned}
& \text {...(1) } X_{t}=\sigma \mathrm{W}_{\text {(st) }}+\mathrm{M} \mathrm{S}_{\mathrm{t}} \\
& \text { اذ تمثل م معلمة الأنتشار diffusion } \\
& \text { drift معلمة الأنحر افة التفار M }
\end{aligned}
$$

Brownian subordinate هي عبارة عن لحركه البراونية ذات الأحداثيات الجزئية W(st)

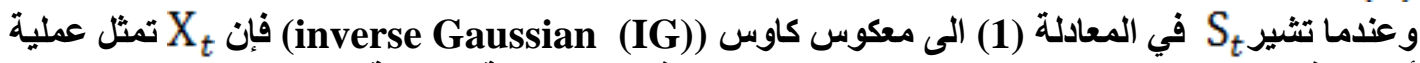

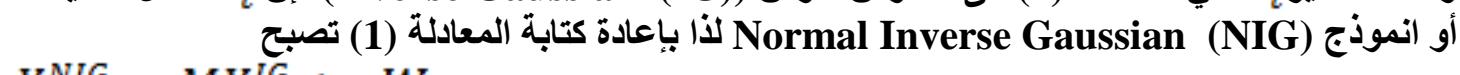
$X_{t}^{N I G}=M X_{t}^{I G}+\sigma W_{X_{t}^{I G}}$

عملية أو إنموذج معكوس كاوس الطبيعي NIG-le'vy ${ }_{t}^{N I G}$ NIG $\left(\alpha, \boldsymbol{\beta}, \boldsymbol{\delta}_{\mathbf{t}}, \mathbf{M t}\right)$ وأن تمثل معكوس عملية معكوس كاوس (Inverse Gaussian) بافتراض أن: $\left.\eta=1, \quad \gamma=\delta \sqrt{\alpha^{2}-\beta^{2}}\right)$

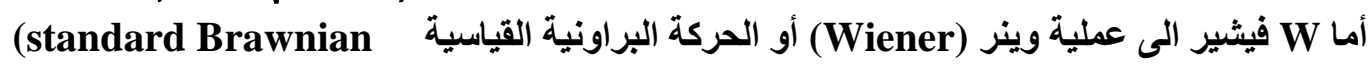
motion)

$$
X_{t}^{N I G}=\beta \delta^{2} X_{t}^{I G}+\delta W_{X}^{I G}
$$
بالرجوع الى المعادلة (2) فإن Xمكن كتابتها بالثكل الأتي:

$$
\begin{aligned}
& \beta=\frac{M}{\sigma^{2}} \\
& \alpha^{2}=\frac{\gamma^{2}}{\sigma^{2}}+\frac{M^{2}}{\sigma^{4}}
\end{aligned}
$$




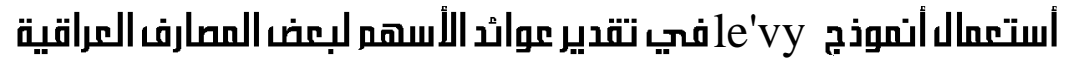

$$
\begin{aligned}
& \gamma=\delta \sqrt{\alpha^{2}-\beta^{2}} \\
& \delta=\sigma
\end{aligned}
$$

$\operatorname{NIG}\left(\alpha, \beta, \delta_{t}\right)$

$$
\text { وإن التوزيع الأحتمالي للأنموذج هو توزيع }
$$

$$
\begin{aligned}
& \text { لذا بالأعتماد على الأفتراضات السابقة يمكن كتابة المعادلة (3) } X_{t}^{N I G} \\
& X_{t}^{N I G}=\beta \delta^{2} X_{t}^{I G}+\delta W_{X}^{I G+M_{t}} \\
& f\left(X_{t}^{N I G}(\chi)\right)=\frac{\alpha \delta_{t}}{\pi} \exp \left[\delta_{t} \sqrt{\alpha^{2}-\beta^{2}}+\beta\left(\chi-M_{t}\right)\right] \times \frac{k_{1}\left(\alpha \sqrt{\delta^{2} t^{2}+\left(\chi-M_{t}\right)^{2}}\right)}{\sqrt{\delta^{2} t^{2}+\left(\chi-M_{t}\right)^{2}}}
\end{aligned}
$$

$$
\alpha>0 \quad, \quad-\alpha<\beta<\alpha-1 \quad, \quad \delta>0
$$

$\mathbf{K}_{\lambda}(u)=\frac{1}{2} \int_{0}^{1} u^{\lambda-1} e^{\frac{-1}{2}\left(z\left(u+u^{-1}\right)\right)} d u$

وان

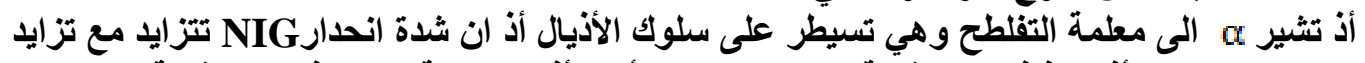

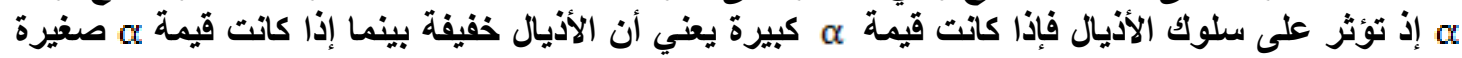

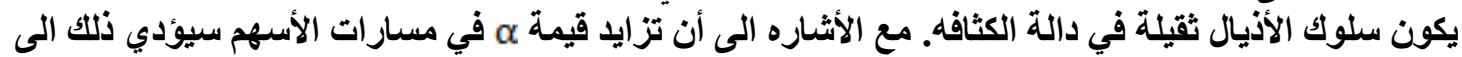
الحا من القفزات الكبيره أما م تثير الى مطلمة الالتواء.

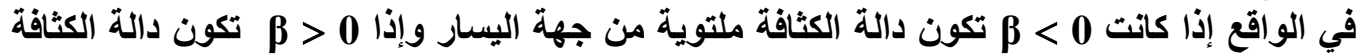

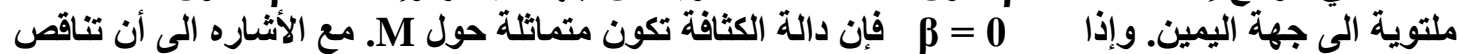

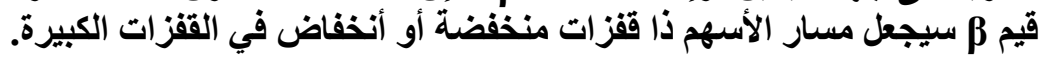
أما $\delta$ تثثير الى معلمة القياس (Scale) وهي مشابه لمعلمة الانحراف المعياري (ه) في التوزيع الطبيعي وهي تمثل مقياس انتثار العو ائدي. Normal Inverse Gaussian 2-2 خصائص انموذج معكوس كاوس الطييعى لعملية ليفى levy (NIG-le'vy) يتميز أنموذج NIG-le'vy بعض الخصائص منها (25,26,17,18,20).

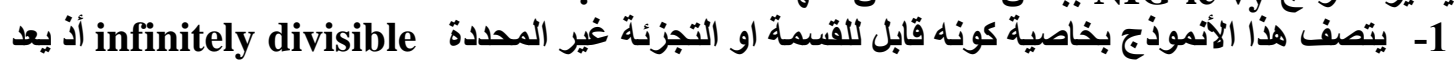

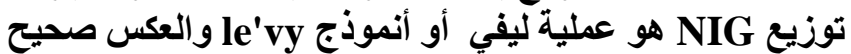

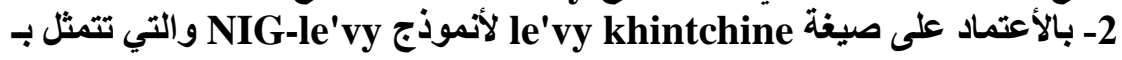




$$
\begin{aligned}
& \Psi(Z)=-\delta t\left(\sqrt{\alpha^{2}-(\beta+Z)^{2}}-\sqrt{\alpha^{2}-\beta^{2}}\right)+M Z \\
& E X_{t}^{N I G}=M_{t}+\frac{\delta t \beta}{\sqrt{\alpha^{2}-\beta^{2}}} \\
& \text { تم أيجاد عزوم انموذج NIG-le'vy اي أن }
\end{aligned}
$$

أـ الأنحراف (drift) لأنموذج NIG-le'vy

$\gamma^{N I G}=\frac{2 \delta \alpha}{\pi} \int_{0}^{1} \operatorname{Sinh}(\beta \chi) k_{1}(\alpha x) d x$

$\operatorname{Sinh}(\beta \chi)=\frac{1}{2}\left(e^{\beta \chi}-e^{-\beta \chi}\right)$

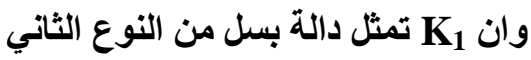

$$
\operatorname{Sinh}(\beta \chi)=\frac{1}{2}\left(e^{\beta \chi}-e^{-\beta \chi}\right)
$$

le'vy بـ مقياس

$$
V^{N I G}(\chi)=\frac{\delta \alpha e^{\beta \chi}(\alpha|x|)}{\pi|x|}
$$

يكون من نوع ذات نشاط غير محدل (infinity activity) ويكون ذات أختلاف او تباين غير محدد

(infinity variaition)

Asset price processes (stock or index)

$$
\text { 3-2 عملية تسعير الأصول (8,21) (الأسهم ، المؤشر) }
$$

إحدى تطييقات عملية ليفي le'vy تتمثل بنمذجة عملية التسعير للأصول (الأسهم او المؤشر) بعملية الزمن

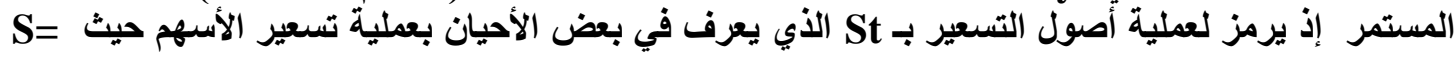

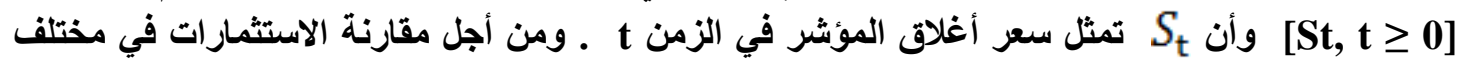
الأوراق المالية ، يكون من الطبيعي النظر الى السعر النسبي المتغير خلال الزمن العو ائد خلاف فترة زمنية واحدة اي يومية هي : 


$$
X_{t}=\log \frac{S_{t}}{S_{t-1}}
$$

$X t=\log S t-\log S t-1$

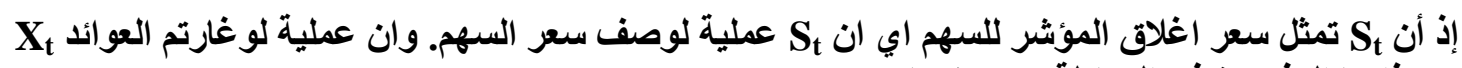

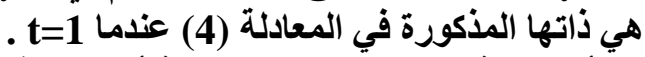
بمأ أن عملية لوغارتم العو ائل اليومية أي t=1 فإن المعادلة (4) تكون كالاتي: $\mathbf{X}^{\mathrm{NIG}}=\boldsymbol{\beta} \delta^{2} \mathbf{X}^{\mathrm{IG}}+\delta W_{X^{I G}}+\mathbf{M}$

$\mathbf{X}^{\mathrm{NIG}} \sim \mathrm{NIG}(\alpha, \boldsymbol{\beta}, \delta, \mathbf{M})$

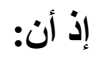

(normal invers Gaussian (NIG))

4-2 طر ائق تقدير معالم إنموذج معكوس كاوس الطييعى تم أستعمال طريقتي العزوم والأمكان الأعظم لأيجاد مقدرات معالم النموذج:

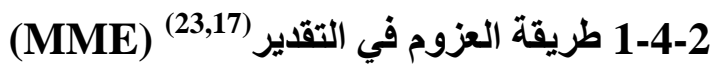

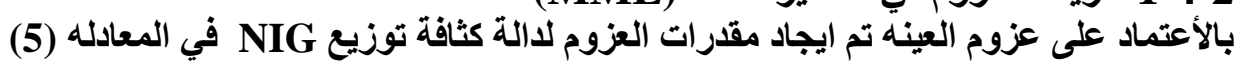
$\hat{\alpha}=\frac{\sqrt{3 b_{2}-4 b_{1}^{2}-9}}{m_{2}\left(b_{2}-5 / 3 b_{1}^{2}-3\right)^{2}}$

$$
\hat{\beta}=\frac{b_{1}}{\sqrt{m_{2}}\left(b_{2}-5 / 3 b_{1}^{2}-3\right)}
$$

$$
\begin{gathered}
\hat{\delta}=\frac{3^{3 / 2} \sqrt{m_{2}\left(b_{2}-5 / 3 b_{1}^{2}-3\right)}}{3 b_{2}-4 b_{1}^{2}-9} \\
\hat{M}=m_{1}-\frac{3 b_{1} \sqrt{m_{2}}}{\left(3 b_{2}-4 b_{1}^{2}-9\right)}
\end{gathered}
$$




$$
\begin{aligned}
& m 1=\frac{1}{n} \sum_{i=1}^{n} x i \\
& m 2=\frac{1}{n} \sum_{i=1}^{n}(x i-m 1)^{2} \\
& b 1=\frac{\frac{1}{n} \sum_{i=1}^{n}(x i-m 1)^{3}}{m 2^{3 / 2}} \\
& b 2=\frac{\frac{1}{n} \sum_{i=1}^{n}(x i-m 1)^{4}}{m 2^{2}}
\end{aligned}
$$

1-1-4-2 خوارزمية لتقير معالم أنموذج معكوس كاوس الطيعى ليفي

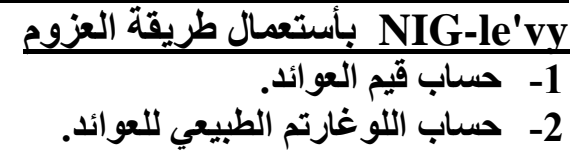

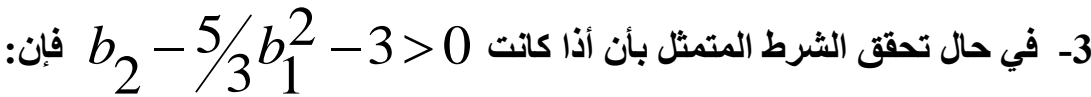
$\hat{\alpha}=\frac{\sqrt{3 b_{2}-4 b_{1}^{2}-9}}{m_{2}\left(b_{2}-5 / 3 b_{1}^{2}-3\right)^{2}}$

$\hat{\beta}=\frac{b_{1}}{\sqrt{m_{2}}\left(b_{2}-5 / 3 b_{1}^{2}-3\right)}$

$\hat{\delta}=\frac{3^{3 / 2} \sqrt{m_{2}\left(b_{2}-5 / 3 b_{1}^{2}-3\right)}}{3 b_{2}-4 b_{1}^{2}-9}$

$\hat{M}=m_{1}-\frac{3 b_{1} \sqrt{m_{2}}}{\left(3 b_{2}-4 b_{1}^{2}-9\right)}$

$\hat{\alpha}=1$

$\hat{\beta}=0$

$\hat{\delta}=0.01$

$\hat{M}=0$ 


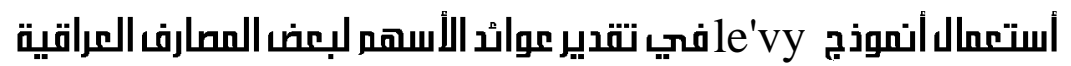

المخطط الأنسيابي لتقدير المعالم الأنموذج بأستعمال مقدرات العزوم-1-1

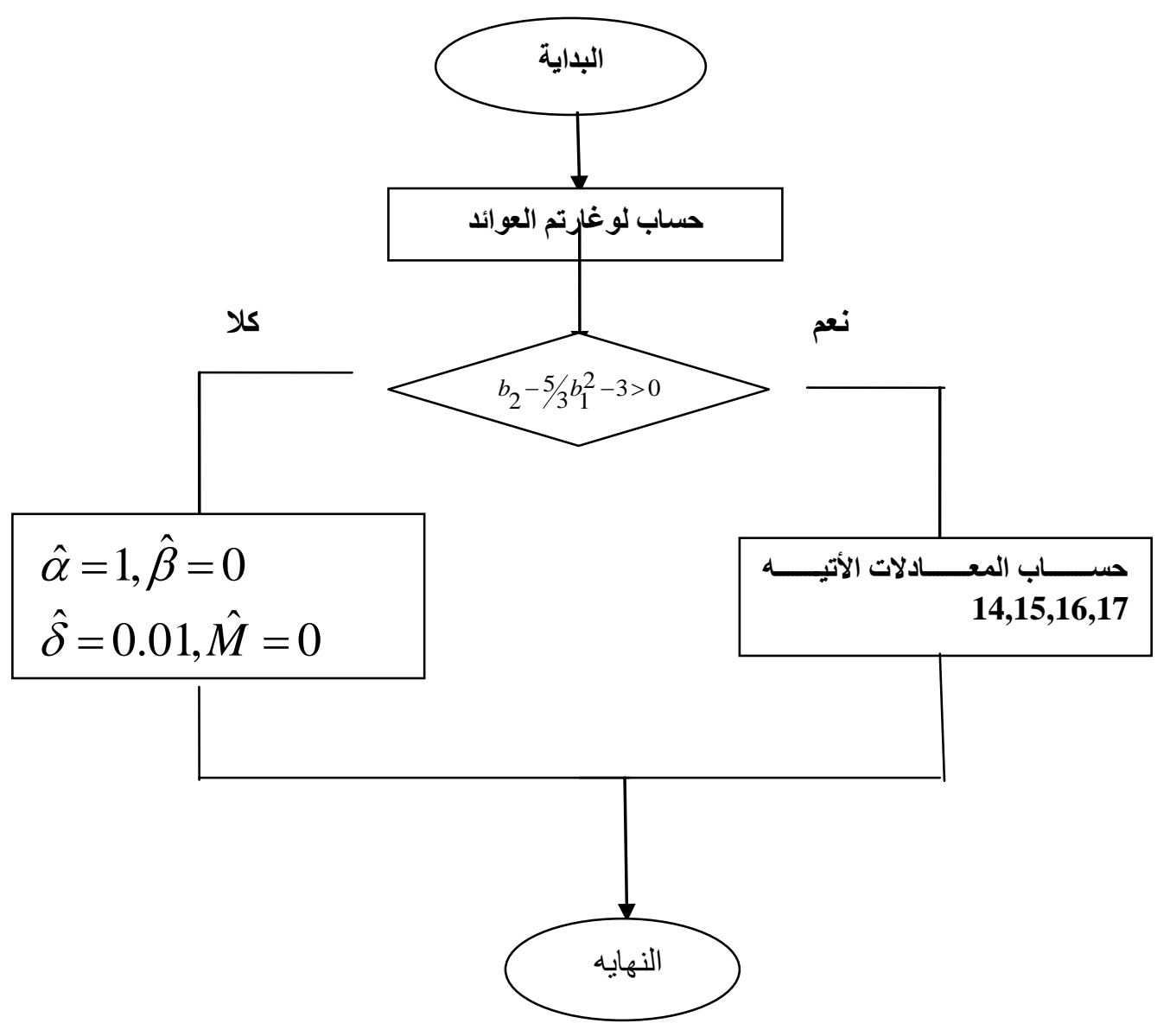

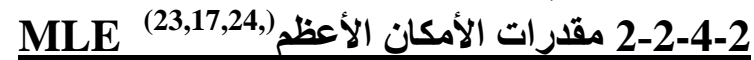

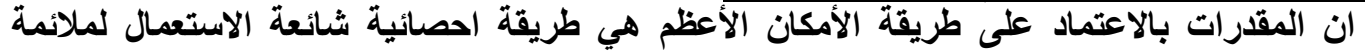

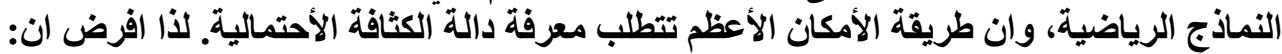

$$
\ell(x, \theta)=\pi_{i=1}^{n} f(x, \theta)
$$

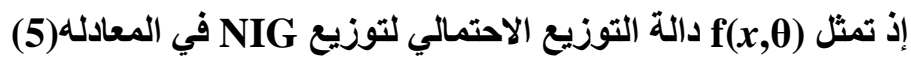

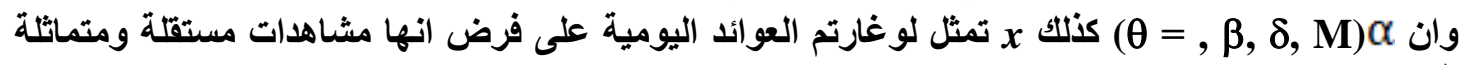
$\mathbf{t}(x, \theta)=\mathbf{n} \ln \frac{\delta \propto}{\pi}+n\left(\delta \sqrt{\alpha^{2}-\beta^{2}}-\beta M\right)+\beta \sum x_{k}$

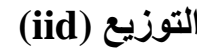

$+\sum_{k=1}^{n}\left(\ln k_{1}\left(\alpha \sqrt{\delta^{2}+\left(x_{k}-M\right)^{2}}\right)-\frac{1}{2} \log \left(\delta^{2}+\left(x_{k}-M\right)^{2}\right)\right)$ 


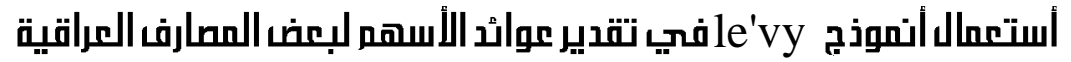

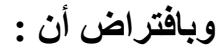

$$
\begin{aligned}
& f=\alpha \sqrt{\delta^{2}+\left(x_{k}-M\right)^{2}} \\
& k_{1}(f)=k_{1}\left(\alpha \sqrt{\delta^{2}+\left(x_{k}-M\right)^{2}}\right) \\
& K_{1}^{\prime}(f)=\left(-\frac{k_{1}(f)}{f}+k_{0}(f)\right)
\end{aligned}
$$

على فرض أن اشتقاق دالة البسل

و وبأثتقاق (1)

$$
\begin{aligned}
& \frac{\partial k_{1}(f)}{\partial \alpha}=-\left(\frac{k_{1}(f)}{f}+k_{0}(f)\right)\left(\frac{\partial f}{\partial \alpha}\right) \\
& \frac{\partial k_{1}(f)}{\partial \beta}=0 \\
& \frac{\partial k_{1}(f)}{\partial \delta}=-\left(\frac{k_{1}(f)}{f}+k_{O}(f)\right)\left(\frac{\partial f}{\partial \delta}\right) \\
& \frac{\partial k_{1}(f)}{\partial M}=-\left(\frac{k_{1}(f)}{f}+k_{0}(f)\right)\left(\frac{\partial f}{\partial M}\right) \\
& \frac{\partial \ell}{\partial \alpha}(\theta)=\frac{n}{\alpha}+\frac{n \alpha \delta}{\sqrt{\alpha^{2}-\beta^{2}}}+\sum_{k=1}^{n}\left(\frac{\frac{\partial k_{1}}{\partial \alpha}}{k_{1}}\right) \\
& \frac{\partial \ell}{\partial \beta}(\theta)=\frac{-n \beta \delta}{\sqrt{\alpha^{2}-\beta^{2}}+\sum_{k=1}^{n}}\left(x_{k}-M\right) \\
& \frac{\partial \ell}{\partial \delta}(\theta)=\frac{n}{\delta}+n \sqrt{\alpha^{2}-\beta^{2}}+\sum_{k=1}^{n}\left(\frac{\partial k_{1}}{\frac{\partial \delta}{k_{1}}-\frac{\delta}{\delta^{2}+\left(x_{k}-M\right)^{2}}}\right) \\
& \frac{\partial \ell}{\partial m}(\theta)=-n \beta+\sum_{k=1}^{n}\left(\frac{\frac{\partial k_{1}}{\delta M}}{k_{1}}+\frac{x_{k}-M}{\delta^{2}+\left(X_{K}-M\right)^{2}}\right)
\end{aligned}
$$

في حالة مساو اتها للصفر لايجاد مقدرات الأمكان الأعظم يكون تعويض المعادلة في الأخرى بصورة

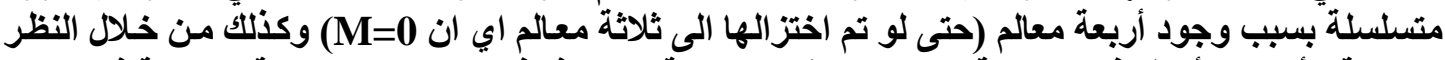

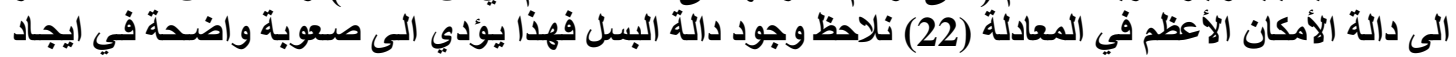

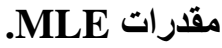


لذا تم استعمال دالة fminsearch في برنامج الماتلاب لغرض الحصول على مقدرات معالم أنموذج بأستعمال الأمكان الأعظم اذ تستعمل هذه الدالة طريقة نيلدر ميا NIG

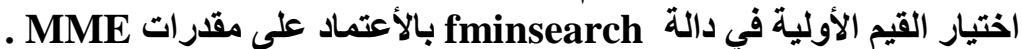

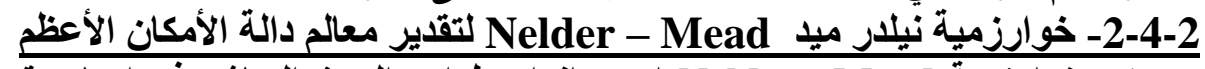

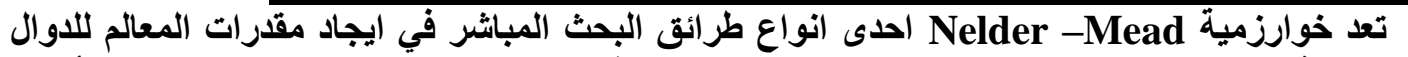

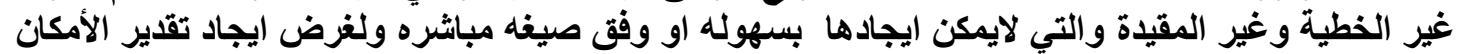

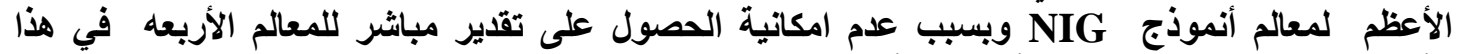

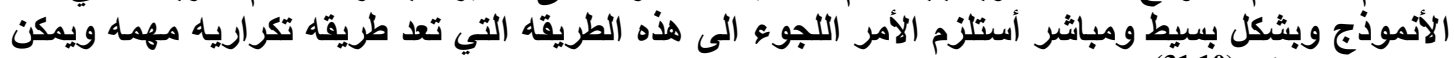
توضيحها كالأتي (31,10)

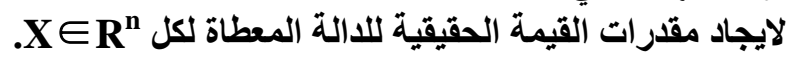

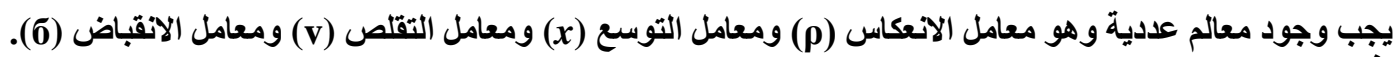
إذان: $\rho>0, x>1, x>\rho, \quad 0<\mathrm{v}<1, \quad 0<0<1$

$\rho=1, x=2, v=1 / 2, \sigma=1 / 2$

وعادة في الاختبار العام تقريباً يستعمل خوارزمية Nelder - Mead

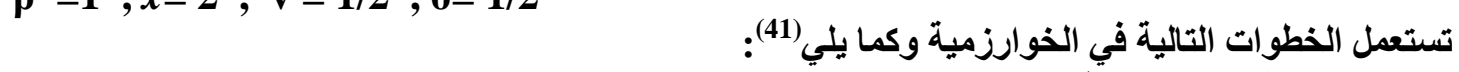
. تمثل متجه النقاط وان X(i) -1

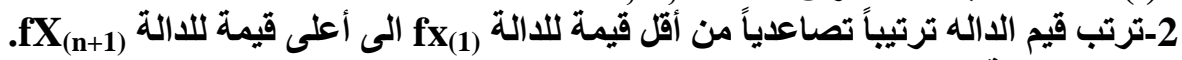

$X r=\bar{X}+\rho\left(\bar{X}-X_{n+1}\right)$ 3-حساب نقطة الانعكاس

$=(1+\rho) \bar{X}-\rho X_{n+1}$

$=2 \bar{X}-X_{n+1} \quad, \bar{X}=\frac{\sum_{i=1}^{n} X i}{n}$

fr=f (xr) ويتم حساب

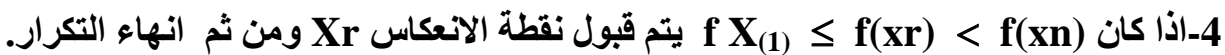

$\mathrm{Xe}=\bar{X}+\boldsymbol{x}(\mathrm{Xr}-\bar{X})$

Xe اذل

$=\bar{X}+\rho x\left(\bar{X}-\mathbf{x}_{\mathbf{n}+1}\right)$

$=\bar{X}+2\left(\bar{X}-\mathbf{X}_{\mathrm{n}+1}\right)$

fe = f (Xe) ثم يتم حساب

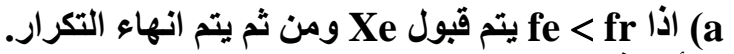

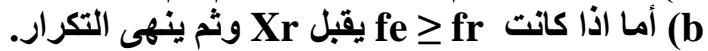

Xr,

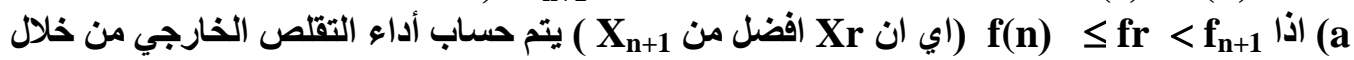
$\mathrm{Xc}=\bar{X}+\mathbf{v}(\mathrm{Xr}-\bar{X})$ $=\bar{X}+1 / 2(\mathrm{Xr}-\bar{X})$

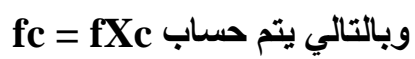

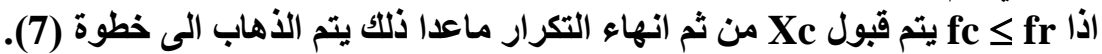

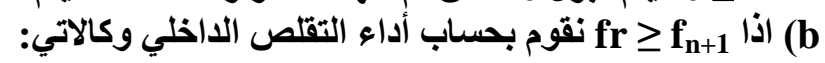




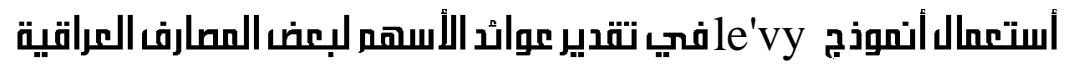

$\mathbf{X}_{\mathrm{cc}}=\bar{X}-\mathbf{v}\left(\bar{X}-\mathbf{X}_{\mathbf{n}+1}\right)$

$=\bar{X}-\left(\mathbf{X}_{\mathrm{n}+1}-\bar{X}\right) / 2$

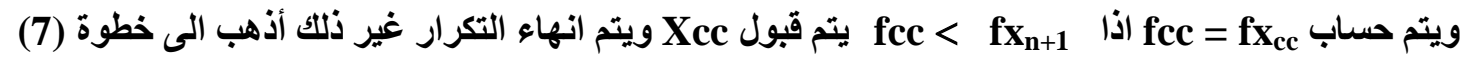

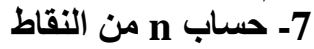

$$
\begin{aligned}
\mathbf{V}(\mathbf{i}) & =\mathrm{X}_{1}+\sigma\left(\mathbf{X}-\mathbf{X}_{1}\right) \\
& =\mathrm{X}_{1}+\left(\mathbf{X i}-\mathbf{X}_{1}\right) / 2
\end{aligned}
$$

$$
\text { ويتم حساب }
$$

$X_{1}, v(2), \ldots . . v(n+1)$

MLE 1-2 مخطط الأنسيابي لتقدير معالم الأنموذج بالأعتماد على مقدرات

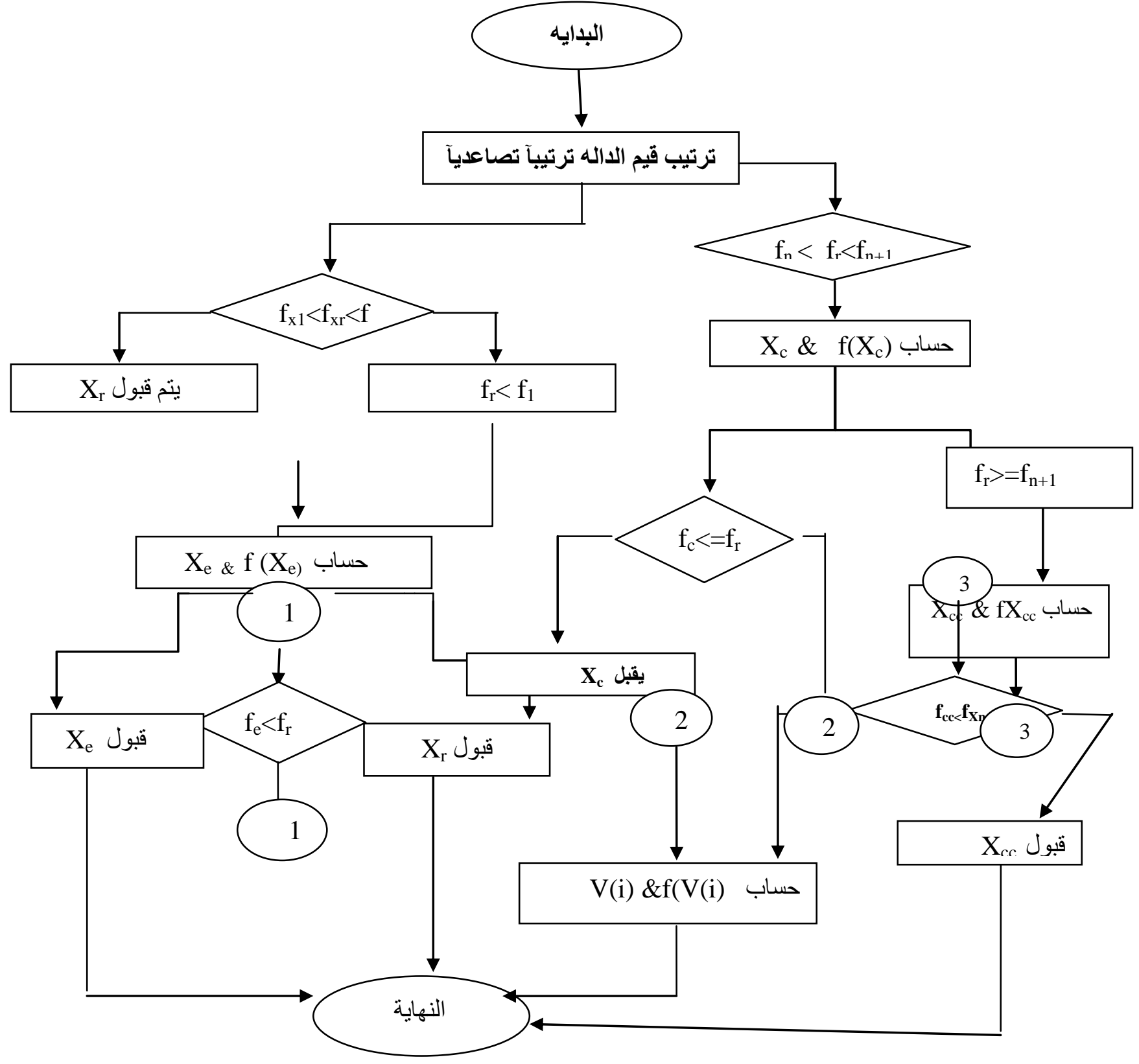

المخطط من اعداد الباحثين 


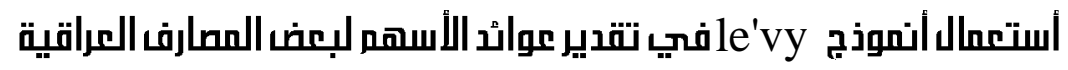

3- الجانب العمطي

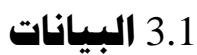

تـم اعتمـاد مجموعة مسن البيانـات لسـر اغـلاق المؤشـر في سوق العـراق لـلأوراق الماليـة لمصـرفي

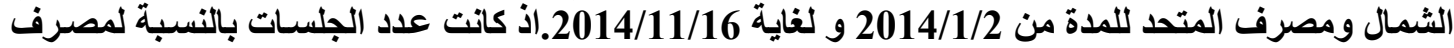
الشـمال (158) جلسـة خـلال عـام التـاول امـا المصرف المتحد فكانت عدد الجلسـات (186) جلسـه خـلال عـام

التداول

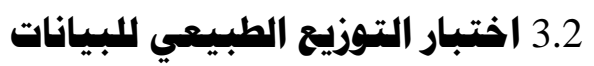

واضح من خلال الجدول الأتي ان البيانات تمتلك قيمة تفرطح عالية وكذلك تكون ملتوية من جهة اليسار . جدول 3.1 يمثل الألتواء والتفرطح لمصرفي الشمال والمتحد

\begin{tabular}{|c|c|c|}
\hline اسم المصرف & التفرطح (b2) & الألتواء (b1) \\
\hline مصرف الشمال & 19.4118 & -2.1215 \\
\hline مصرف المتحد & 10.7332 & --0.7002 \\
\hline
\end{tabular}

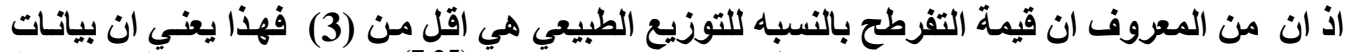

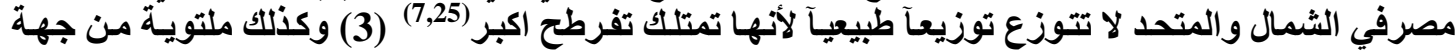
اليسار.

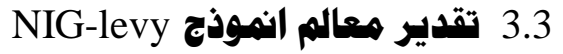

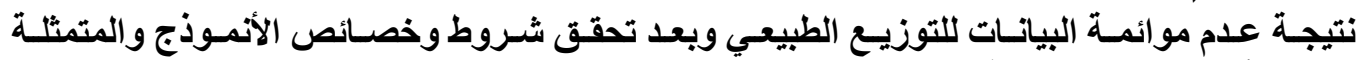

بالأستقلاليه والأستقراريه الموضحة في الثكل (3.1a,3.1b) شكلa|3.1 ) يمثل استقراريه واستقلاليه العوائد لمصرف الثمال

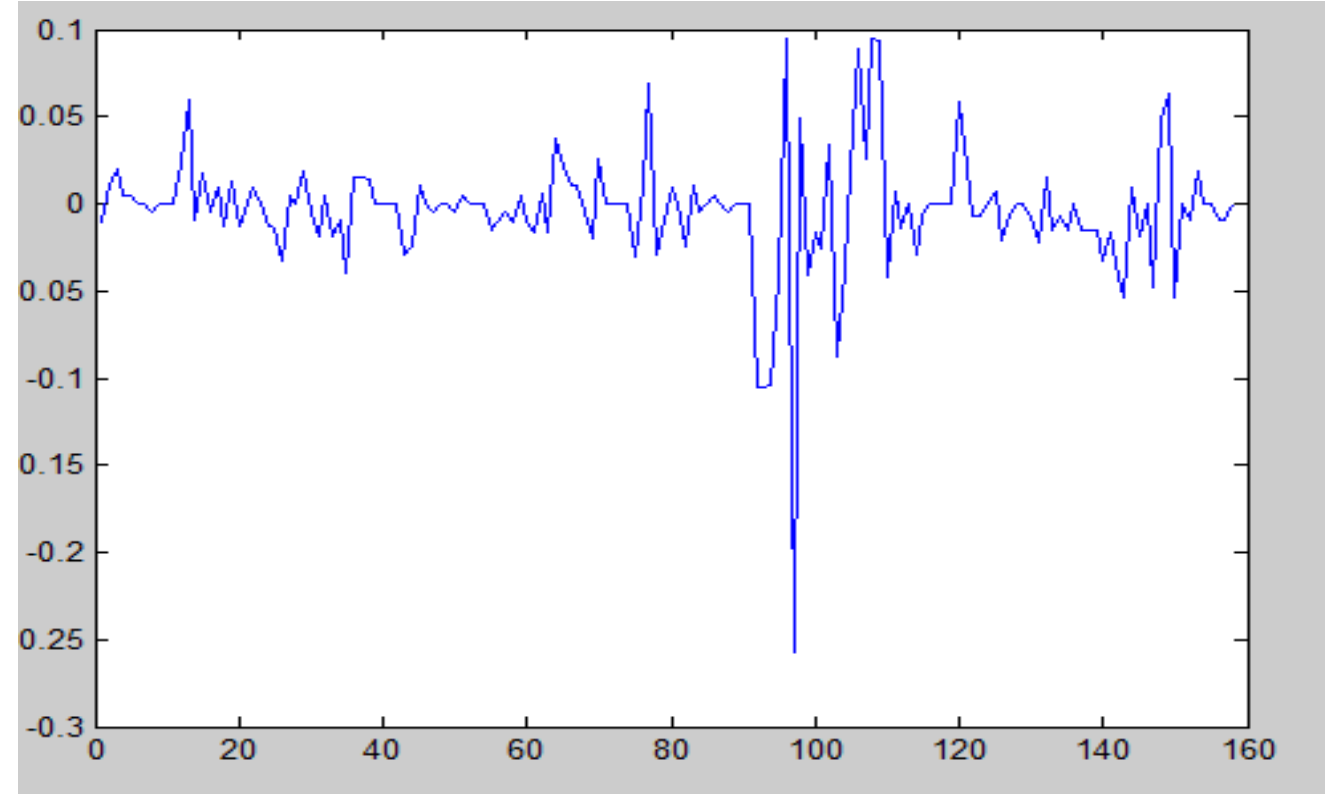




\section{استممالا اتموزي}

شكلb|(3.1 ) يمثل استقراريه واستقلاليه العوائد لمصرف المتحد

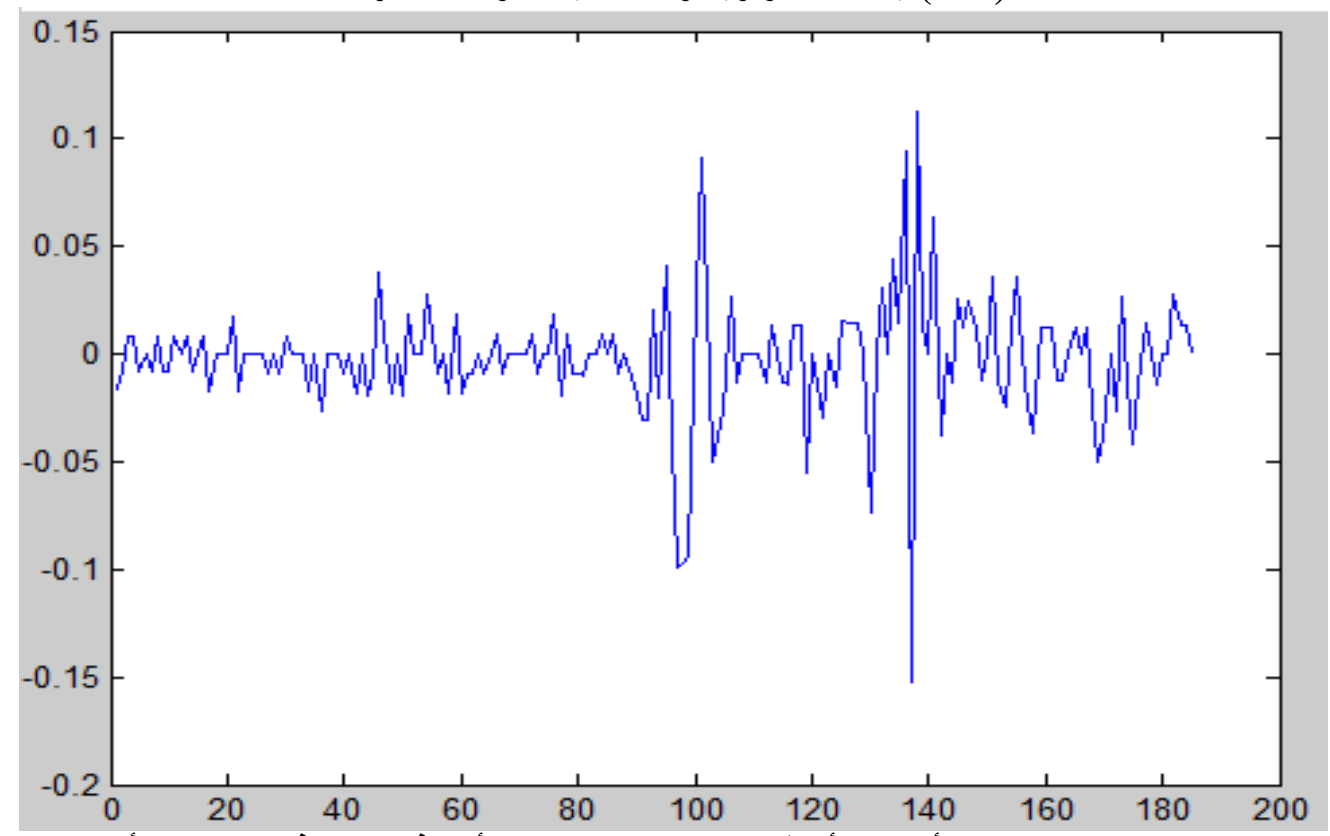

لذا تم استعمال مقدرات العزوم و الأمكان الأعظم لغرض تقلير معالم الأنموذج وكما في الجدول الأتي.

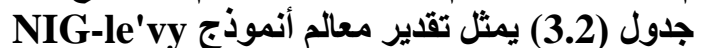

\begin{tabular}{|c|c|c|c|c|c|}
\hline & الطريقة & $\alpha$ & B & $\delta$ & M \\
\hline \multirow[t]{2}{*}{ مصرف الثمال } & MME & 17.4866 & -6.683 & 0.0178 & -0.003 \\
\hline & MLE & 7.3753 & -1.5577 & 0.0103 & -0.0017 \\
\hline \multirow[t]{2}{*}{ مصرف المتحد } & MME & 23.9094 & $\begin{array}{l}-3.6572 \\
\end{array}$ & 0.0181 & -0.000162 \\
\hline & MLE & 15.6324 & -2.6351 & 0.0121 & -0.0005 \\
\hline
\end{tabular}

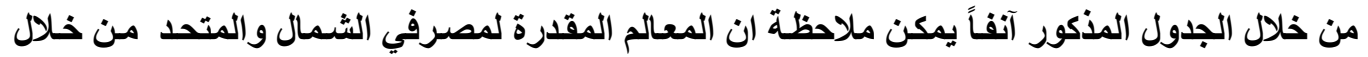

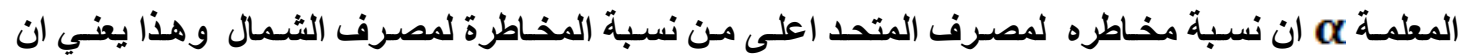

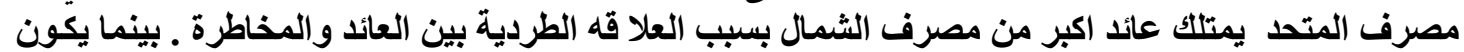

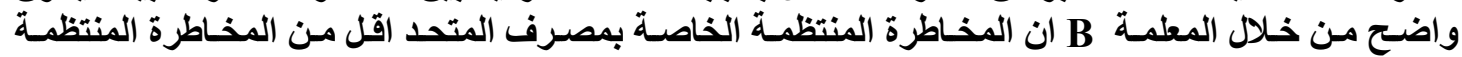

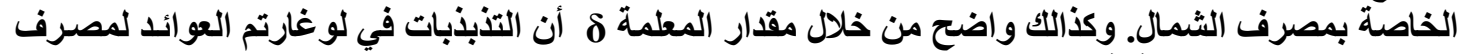

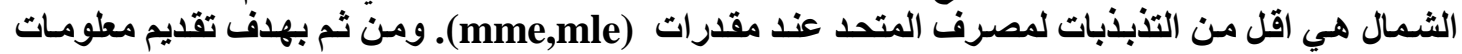

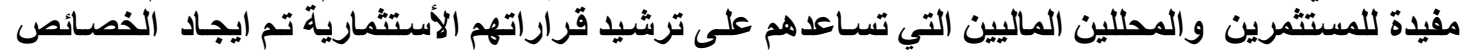
الأحصائيه لأنموذج مIG-levy في جدول (3.3) الأتي 
جدول (3.3 ) يمثل الخصائص الاحصائية لأنموذج NIG - le'vy

\begin{tabular}{|c|c|c|c|c|c|c|c|}
\hline & & Mean & Var & Sek & Kurt & $\overline{C . V}$ & drift \\
\hline \multirow{2}{*}{ مصرف } & MME & -0.004 & 0.0013 & -2.1223 & 19.4236 & -3.4797 & -0.0040 \\
\hline & MLE & -0.0039 & 0.0015 & -2.3253 & 50.6121 & -9.8511 & $\begin{array}{c}-0.0039 \\
\end{array}$ \\
\hline \multirow{2}{*}{ مصرف المتحد } & MME & $\begin{array}{c}-0.0029 \\
\end{array}$ & $7.7717 \mathrm{e}^{-04}$ & -0.7008 & 10.7468 & -9.6204 & -0.0029 \\
\hline & MLE & -0.0026 & $8.082 \mathrm{e}-04$ & -1.1712 & 20.9193 & -11.065 & -0.0026 \\
\hline
\end{tabular}

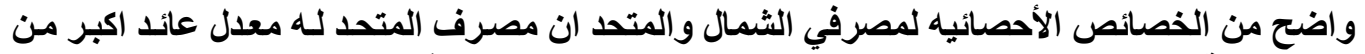

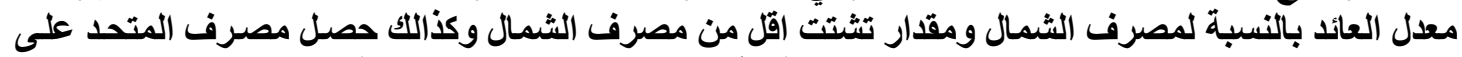

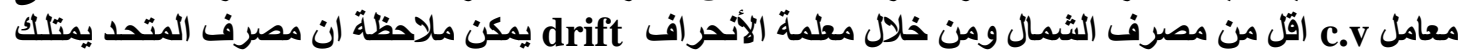

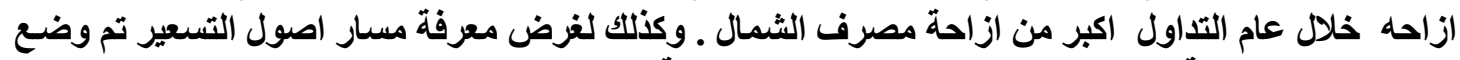

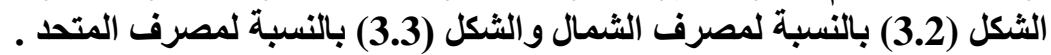

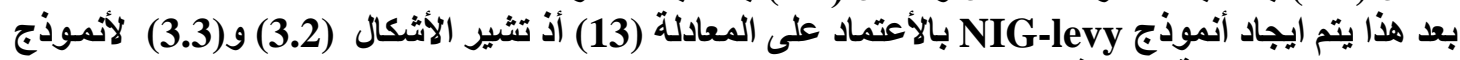

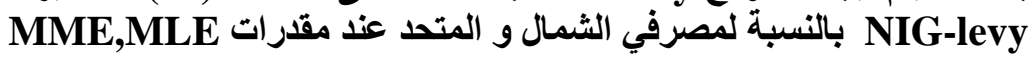

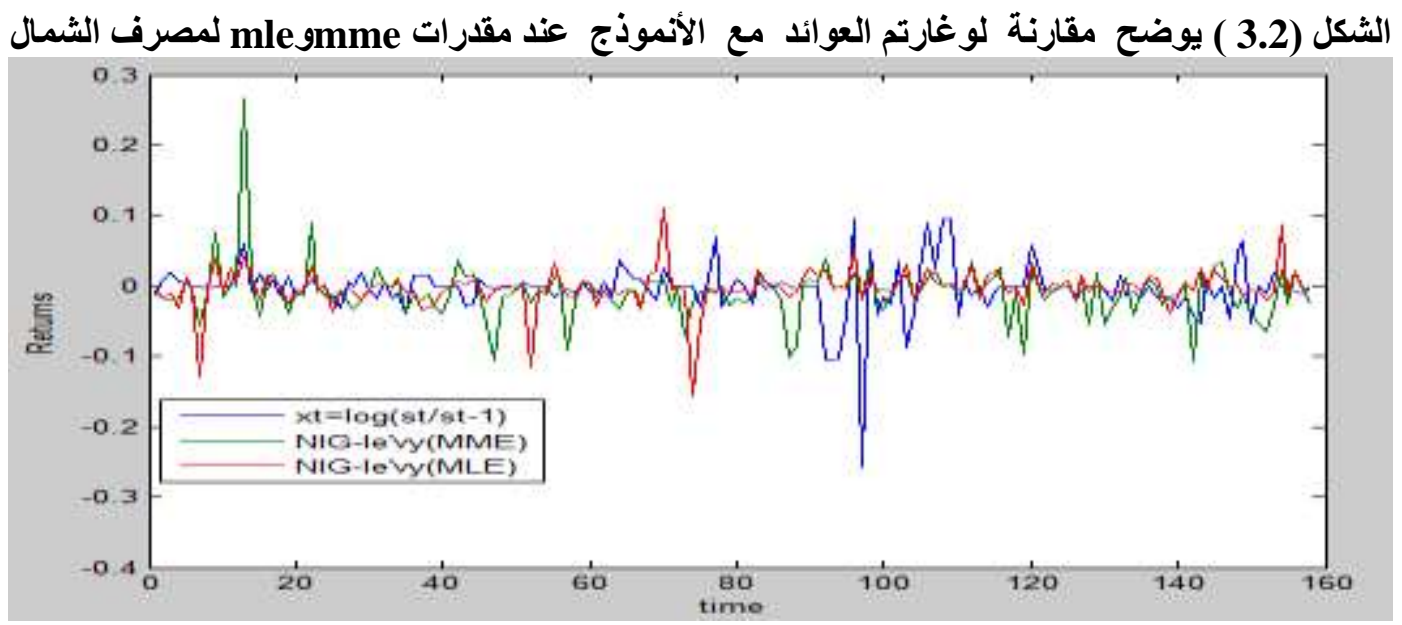

الشكل ( 3.3) يوضح مقارنة لوغارتم العوائد مع الأنموذج عند مقرات mleوmme لمصرف المتحد

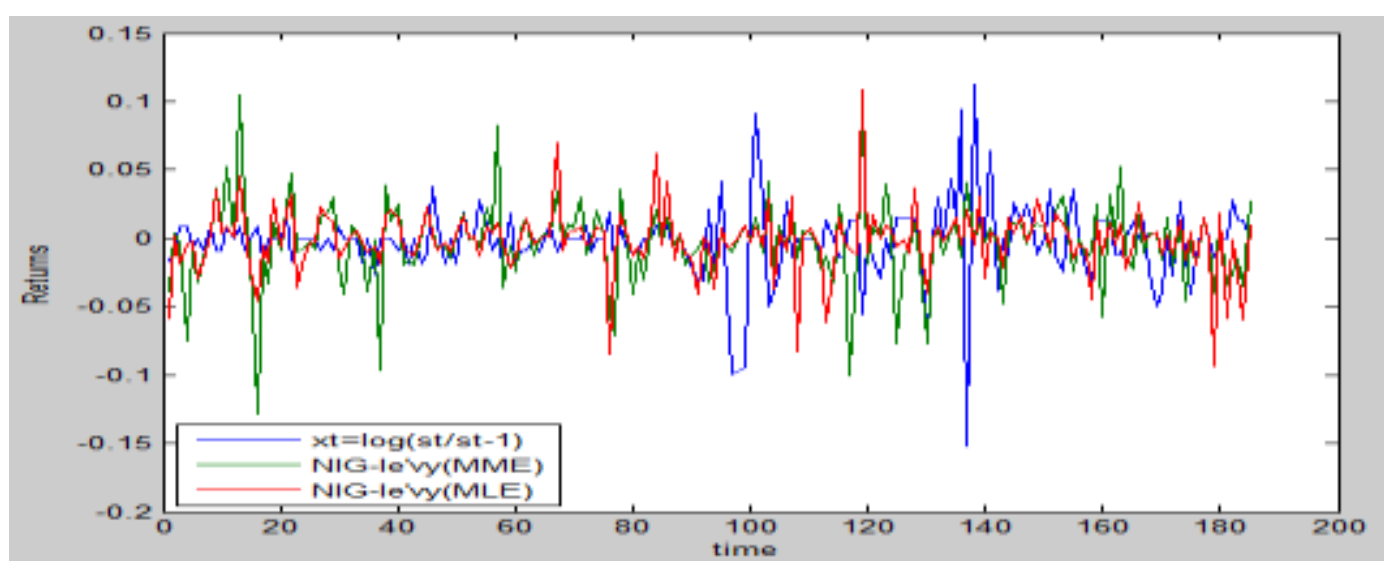




\section{استمصال انموذج}

من خلال الثكل (3.2), (3.2) ان مقدرات MLE أقرب الى بيانات لوغارتم العو ائد من مقدرات

M و هذا واضح من خلال أيجاد MSE للأنموذج عند مقدرات MME,MLE في الجدول الأتي.

جدول (3.4) يمثل متوسط مجموع مربعات الخطأ لأنموذج NIG-le'vy

\begin{tabular}{|c|c|c|}
\hline & & Mse \\
\hline \multirow{2}{*}{ الثمرف } & MME & 0.0014 \\
\hline & $\overline{\text { MLE }}$ & $\overline{0.000717}$ \\
\hline \multirow{2}{*}{ مصرف } & MME & $\begin{array}{l}0.000844 \\
\end{array}$ \\
\hline & MLE & 0.000548 \\
\hline
\end{tabular}

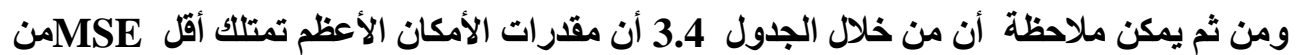

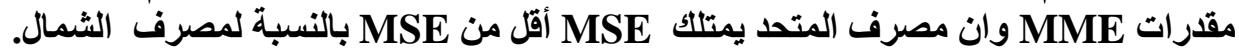

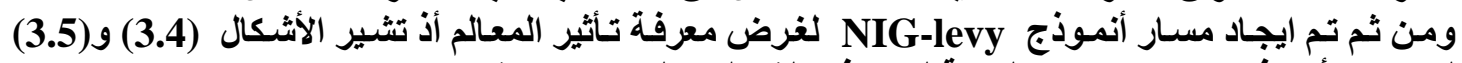

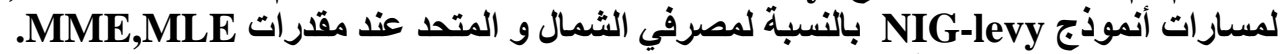
الثكل(3.4) يوضح مقارنة مسار لوغارتم العوائد مع مسار الأنموذج عند مقدرات mme و mle لمصرف الثمال

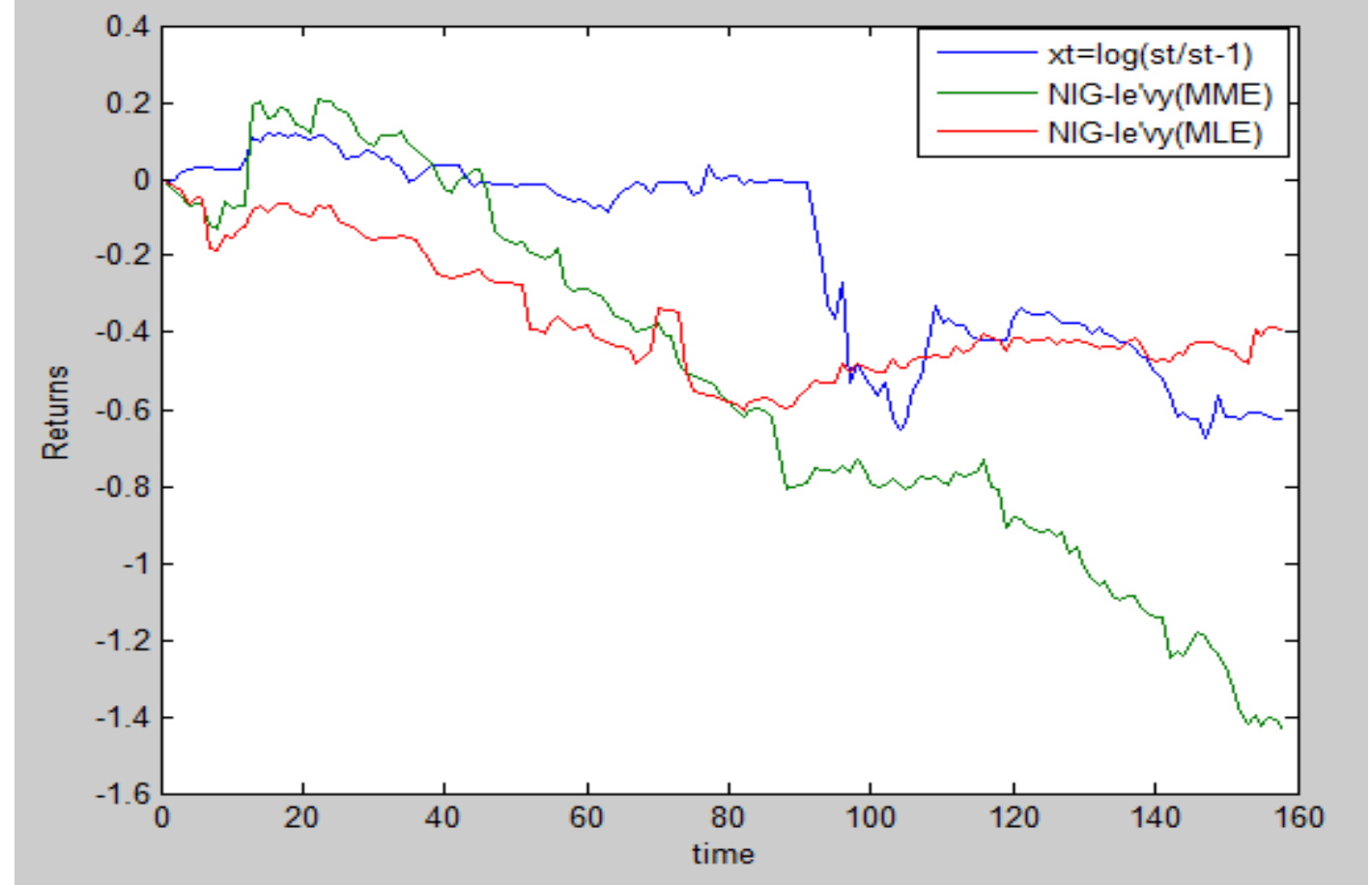


الثكل( 3.5) يوضح مقارنة مسار لوغارتم العوائد الفعليه مع مسار الانموذج عند مقدرات mleوmme لمصرف المتحد

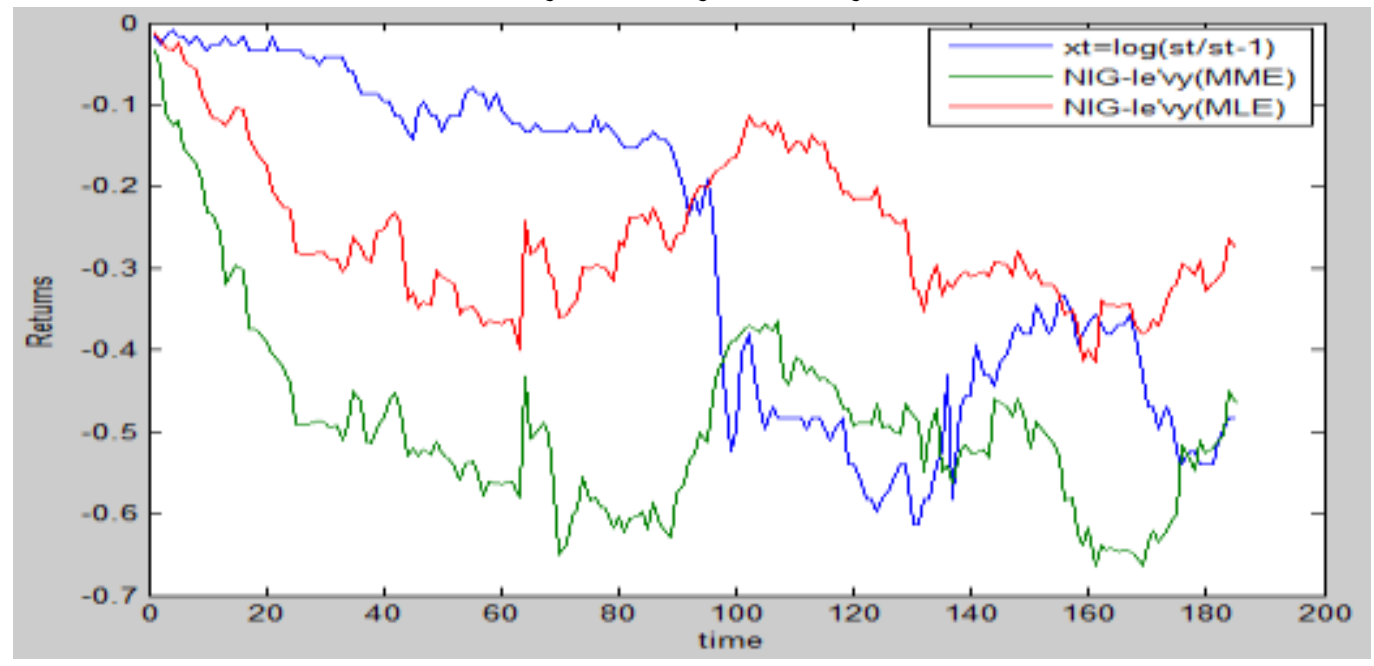

ومن الأشكال (3.4) و(3.5) يلاحظ ان مسار مصرف المتحد عند مقدرات MME,MLE يكون ذا لألات

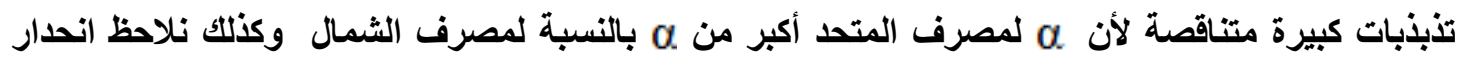

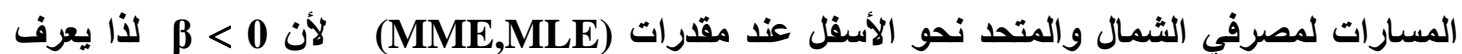

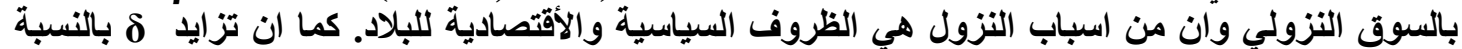

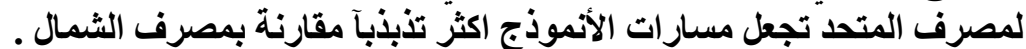

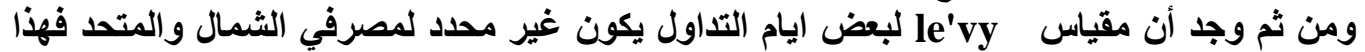

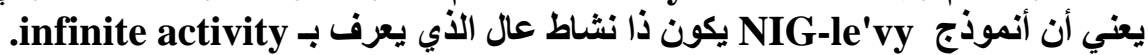

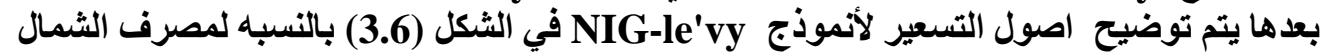

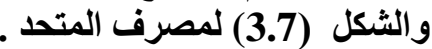
الثكل (3.6) يوضح مقارنة القيمة الفعلية لمصرف الثمال مع انموذج اصول التسعير (القيمة الدقيقية) عند

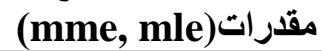

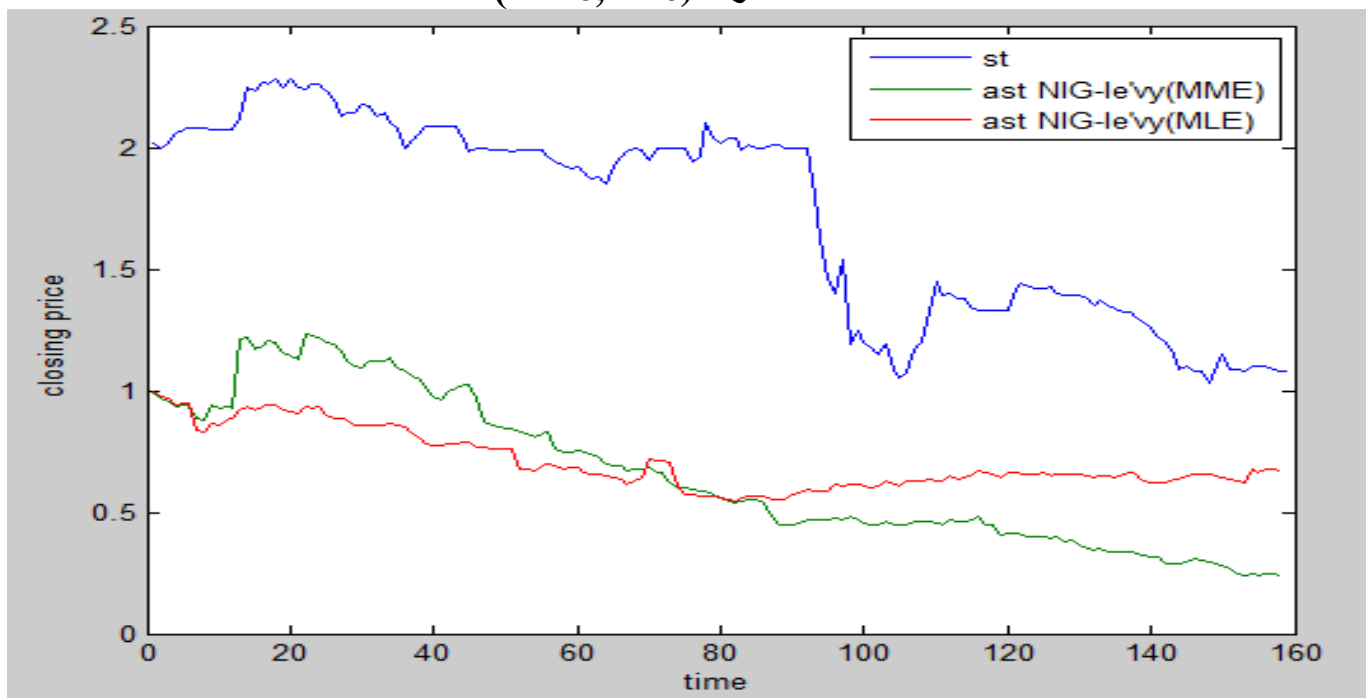




\section{استممالا انموزي}

الشكل (3.7) يوضح مقارنة القيمه الفعليه لمصرف المتحد مع انموذج اصول التسعير(القيمه الحقيقيه) عند

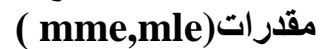

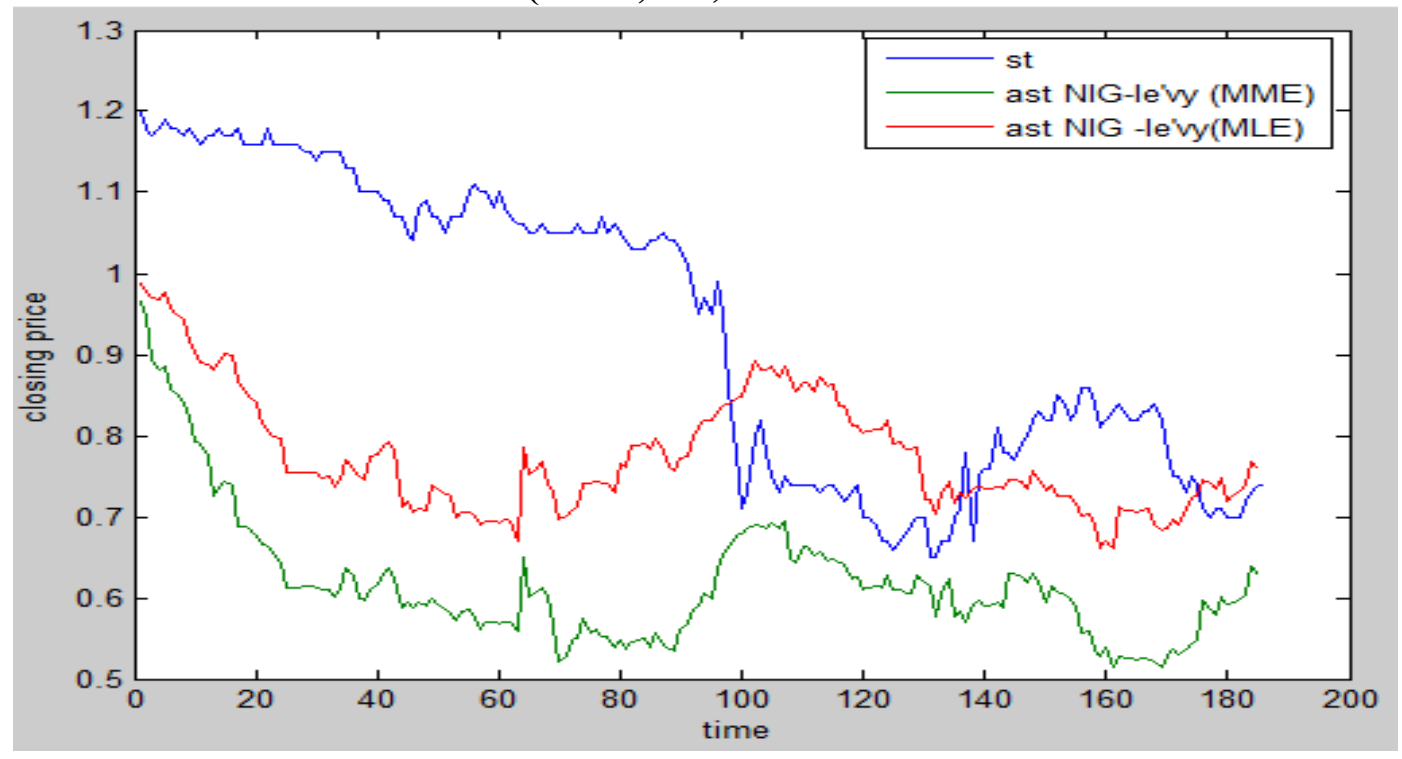

4-الأستنتاجات

من خلال توظيف الطرائق التي تم تقديمها في الجانب النظري على بياتات سعر أغلات التاق المؤشر لعام

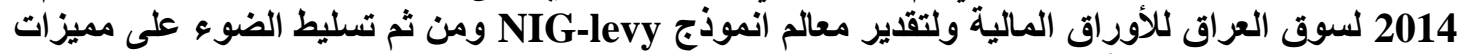

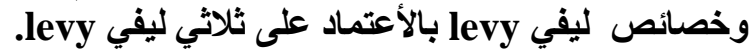

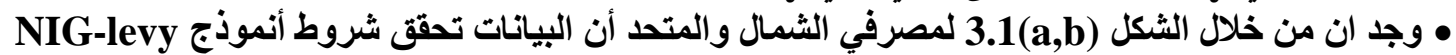

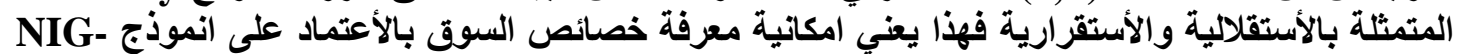

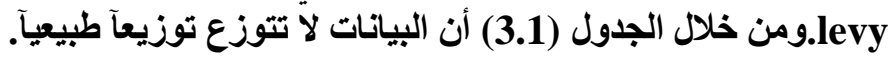

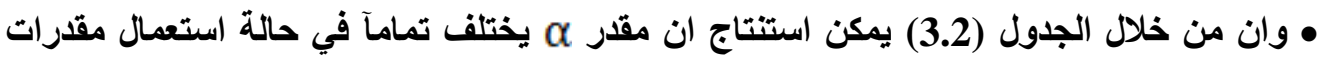
الأمكان الأعظم ومقدرات العزوم وكنلك في حال استعمال التكرار ات ات العالية.

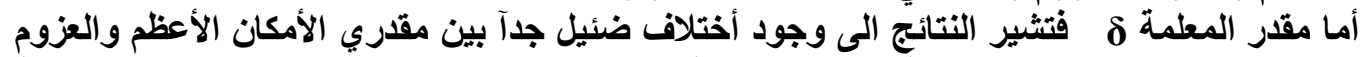

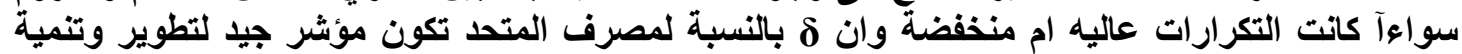

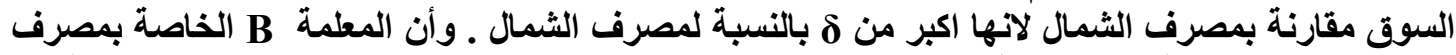

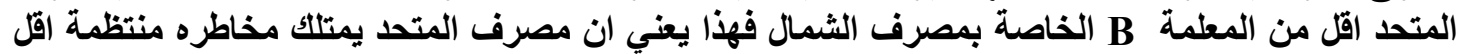

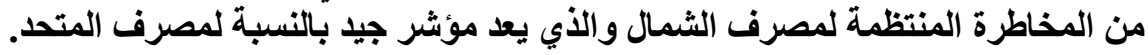

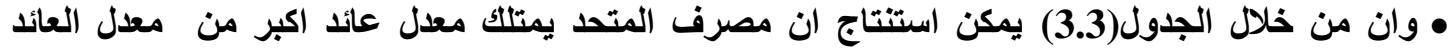

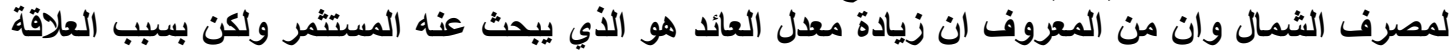

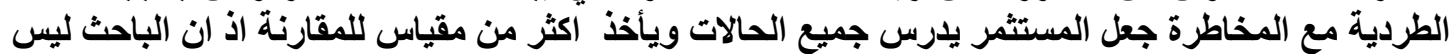

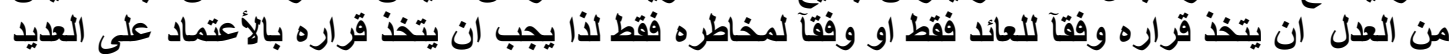

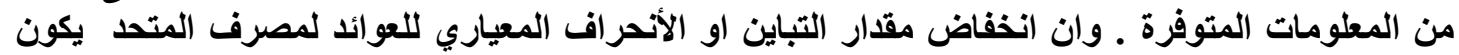

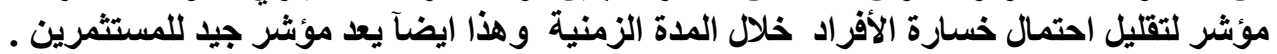

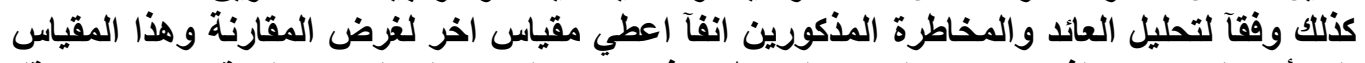

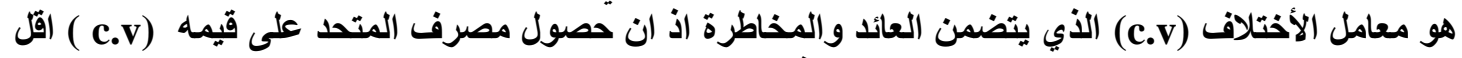
من (c.v) لمصرف الثمال يجعل مصرف المتحد افضل للمستثمر عند مقدرات (mme,mle). 
وان مقدار الأزاحه بالنسبة لمصرف المتحد اكبر من مقار الأزاحه لمصرف الثمال عند مقدرات خلال عام التداول. (mme,mle)

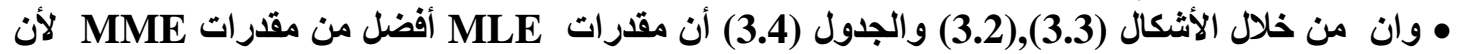

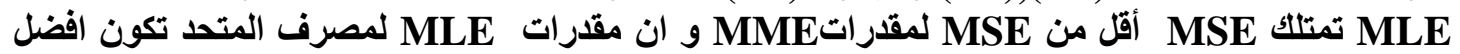

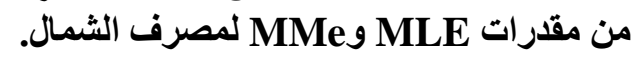
• ولغرض مقارنة قيم أصول التسعير لمصرفي المتحد والثمال عند مقرات (mme,mle) لكل يوم تداول

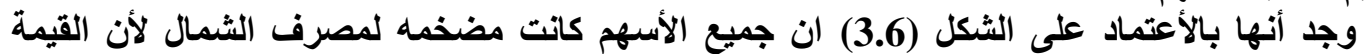

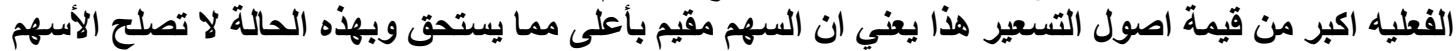

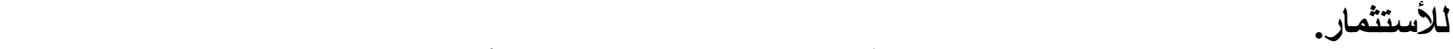

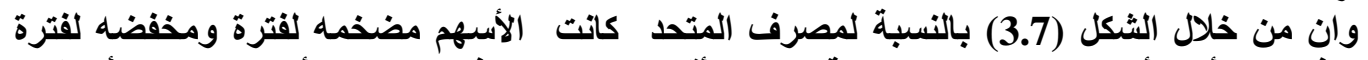

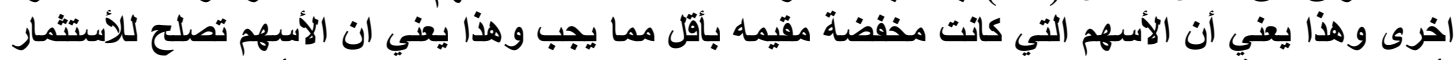

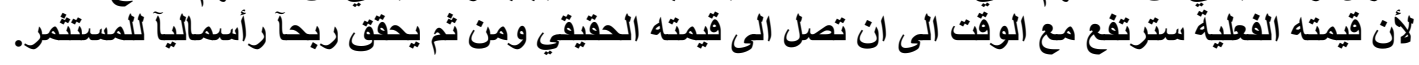
1- حطاب سامي ،2007،المحافظ الأستثماريه ومؤشرات أسعار الأسهم وصناديق الأستثمار ، بدعوه من هيئة الأوراق الماليه والميلع ابوظبي.

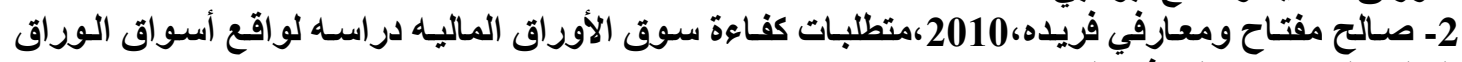
الماليه العربيه وسبل رفع كفاعتهاريا.

3- Agbawo chidinma,2010,Model Risk for Barrier option when priced under different le'vy Dynamics, university of Stellenbosch stochastic calculus ,university of Sheffield.

4- Baudin Michael \&Bihorel sebastien ,2015, package nelder-mead .compution of the unconstrained minimum of fminsearch function .with the Nelde -mead alagorithm, <sb.pmlab@gmail.com>

5- Cont Rama ,2001,Empirical properties of asset returns :stylized facts and statistical issues, Web address: http://www.cmap - polytechnique .fr / rama, QUANTITATIVE FINANCE VOLUME 1,p.p (223-236).

6-Cont Rama \& and Tankover Perter ,2004,Finanical modeling with jump processes , University of Cambridge\& University of Maryland, , A CRC Press Company Boca Raton London New York Washington, D.C..

7- Fernandes Jorge ,2012, fitting the Normal Inverse Gaussian distribution to the s\&p 500 stock return data, Dept. of Mathematics U Mass Dartmouth Dartmouth MA 02747

Email: Jfernandes7@umassd.edu.

8- Goncu Ahmet \& Karahan Oguz Mehmet \& Kuzubas Umut Tolga ,2015, A comparative Goodness- of - fit Analysis of Distribution of some le'vy processes and Heston model to stock index Returns ,Xian Jiaotong Liverpool university \&Bogazi ci university, Center for Economics and Econometrics, Istanbul, Turkey.

9 -Hakwa Brice ,2011, Phd seminar : normal Inverse Gaussian process for commodities modeling and Risk -management ,Bergische university, Fachbereich Angewandte Mathematik - Stochastik Hakwa@math.uniwuppertal.de. 


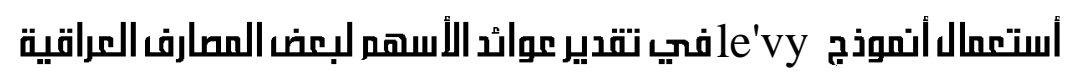

10-Hainaut Donatien ,2011,Financial modeling with switching le'vy processes, ESC Rennes Business School \& CREST, France.

Email: donatien.hainaut@esc-rennes.fr .

11 - Kitchen cliff ,2009,normal Inverse Gaussian (NIG) process with Application in Mathematical Finance, university of Calgary, The Mathematical and Computational Finance Laboratory.

12 - Kou G.s ,2007,levy processes in asset pricing, Columbia University .

13- Lagarias Jeffrey \&Reeds James \& WRIGHT MARGARET H.\& WRIGHT PAUL E. ,1988,Convergence properties of the Nelder-mead simplex method in law dimensions, Society for Industrial and Applied Mathematics, Vol. 9, No. 1, pp. 112-147

\section{http://www.siam.org/journals/siopt/9-1/30347.html}

14-Lin Rong ,2011, An introduction le'vy processes : definitions \& properties

15- Mastro Michael ,2013, financial derivative and Energy market valuation theory and implementation in matlab ,John wiley \&sons, http://www.willey.com/go/permission

16-Papapautolen Atonis ,2008, An introduction to le'vy processes with applications in finance, university of Piraeus.

17-Nielsen Barndorff ,1998,processes of NIG type, Department of Mathematical Sciences, University of Aarhus, Finance Stochast. 2, p.p 41-68.

18- Nielsen Barndorff \& Shephard Neil,2000, modeling by le'vy processes for financial Econometrics, University of Aarhus \& University of Oxford .

19- Ouwehand .W \& West Lydia ,2012,American monte carlo option pricing under pure Jump le'vy models, Copyright $\odot$, university of Stellenbos.

20- Reccion maria \&Ma chiar \& Deville Damien ,2008,on le'vy processes for option pricing :Numerical Methods and calibration to Index options ,university of politecnica Delle Marche, Anno Accademico.

21- Ribeior Claudia \&Wobber Nick ,2003, A monte Carlo method for the Normal Inverse Gaussian option valuation model using an Inverse Gaussian Bridg, City University, Cass Business School

London EC2Y 8HB.

22- Robbertse Lodewickes Johannes ,2006, on the modeling of asset Resturns and the calibration of Eurobean option pricing models, university of Johannesburg.

23-Saebo karsten ,2009,pricing Exotic option with normal inverse Gaussian market model using numerical path Integration ,Department of Mathematical sciences Norwegian university of science and Technology.

24- Teneng Dean,2013,NIG le'vy process in asset price modeling case of Estonion companies ,Institue of mathematical statistic ,university of Tartu, MPRA Paper No. 47852 p.p 891-896.

25-Teneng Dean,2013,A note on NIG-le'vy process :in asset price Modeling :case of Estonian companies , Institue of mathematical statistic ,university of Tartu, MPRA Paper No. 47862. 


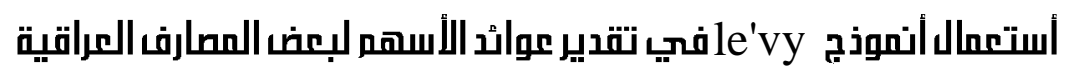

\section{Use the le'vy Model on stock returns for some Iraqi banks estimate}

\section{Abstract}

In this article we study a single stochastic process model for the evaluate the assets pricing and stock.,On of the models le'vy . depending on the so-called Brownian subordinate as it has been depending on the so-called Normal Inverse Gaussian (NIG). this article aims as the estimate that the parameters of his model using my way (MME,MLE) and then employ those estimate of the parameters is the study of stock returns and evaluate asset pricing for both the united Bank and Bank of North which their data were taken from the Iraq stock Exchange.

which showed the results to a preference MLE on MME based on the standard of comparison the average square error (MSE) .

and the yield rate of the stock of the Bank United is higher than the rate of returns for the North Bank as well as the United owning less coefficient c.v compared with the North Bank and both estimater (MME,MLE) .therefore the United Bank is the best investment of the Northa Bank in addition, the North Bank was less efficient than the United Bank for, leading this speech to preference of investors to invest with united Bank and its superiority on the North Bank.

Key words: Brownian motion, subordinate, Brownian subordinate, Normal Inverse Gaussian model (NIG) 